\title{
Risk factors for determining length of intensive care unit and hospital stays following correction of cervical deformity: evaluation of early severe adverse events
}

\author{
Presented at the 2020 AANS/CNS Joint Section on Disorders of the Spine and Peripheral Nerves \\ Rushikesh S. Joshi, BS, ${ }^{1}$ Darryl Lau, MD, ${ }^{1}$ Alexander F. Haddad, BS, ${ }^{1}$ Vedat Deviren, MD, ${ }^{2}$ and \\ Christopher P. Ames, MD ${ }^{1}$
}

Departments of ${ }^{1}$ Neurological Surgery and ${ }^{2}$ Orthopedic Surgery, University of California, San Francisco, California

\begin{abstract}
OBJECTIVE Correction of rigid cervical deformities can be associated with high complication rates and result in prolonged intensive care unit (ICU) and hospital stays. In this study, the authors aimed to examine the risk factors contributing to length of stay (LOS) in both the hospital and ICU following adult cervical deformity (ACD) surgery and to identify severe adverse events that occurred in this setting.
\end{abstract}

METHODS A retrospective review of ACD patients who underwent posterior-based osteotomies for deformity correction from 2010 to 2019 was performed. Inclusion criteria were cervical kyphosis $>20^{\circ}$ and/or cervical sagittal vertical axis (cSVA) $>4 \mathrm{~cm}$. Multivariate analysis was used to identify risk factors independently associated with ICU and hospital LOS.

RESULTS A total of 107 patients were included. The mean age was 63.5 years, and $61.7 \%$ were female. Over half (52.3\%) underwent 3 -column osteotomies, while $47.7 \%$ underwent posterior column osteotomies. There was significant correction of all cervical parameters: cSVA ( $6.0 \mathrm{vs} 3.6 \mathrm{~cm}, p<0.001)$, cervical lordosis $\left(8.2^{\circ} \mathrm{vs}-5.3^{\circ}, p<0.001\right)$, cervical scoliosis $\left(6.5^{\circ}\right.$ vs $\left.2.2^{\circ}, p<0.001\right)$, and T1-slope $\left(40.2^{\circ}\right.$ vs $\left.34.5^{\circ}, p<0.001\right)$. There were also reciprocal changes to the distal spine: thoracic kyphosis ( $54.4^{\circ}$ vs $46.4^{\circ}, p<0.001$ ), lumbar lordosis $\left(49.9^{\circ}\right.$ vs $45.8^{\circ}, p=0.003$ ), and thoracolumbar scoliosis $\left(13.9^{\circ}\right.$ vs $\left.11.1^{\circ}, p=0.009\right)$. Overall, 4 patients $(3.7 \%)$ suffered aspiration-related complications, 3 patients $(2.8 \%)$ experienced dysphagia requiring a feeding tube, and 4 patients (3.7\%) had compromised airways, with 1 resulting in death. The mean ICU and hospital LOS were 2.8 days and 7.9 days, respectively. Multivariate analysis identified three factors independently associated with longer ICU LOS: female sex (3.0 vs 2.4 days, $p=0.004$ ), $\geq 12$ segments fused ( 3.5 vs 1.9 days, $p=0.002$ ), and postoperative complication ( 4.0 vs 1.9 days, $p=0.017$ ). These same factors were independently associated with longer hospital LOS as well: female sex ( 8.3 vs 7.3 days, $p=0.013$ ), $\geq 12$ segments fused (9.4 vs 6.2 days, $p=0.001$ ), and complication (9.7 vs 6.7 days, $p=0.026$ ).

CONCLUSIONS Posterior-based osteotomies are very effective for the correction of ACD, but postoperative hospital stays are relatively longer than those following surgery for degenerative disease. Risk factors for prolonged ICU and hospital LOS consist of both nonmodifiable (female sex) and modifiable ( $\geq 12$ segments fused and presence of complication) risk factors. Additional multicenter prospective studies will be needed to validate these findings.

https://thejns.org/doi/abs/10.3171/2020.6.SPINE20826

KEYWORDS cervical; intensive care unit; length of stay; osteotomy; deformity

$\mathrm{O}$ VER the last several years, there has been an increase in the understanding of adult cervical deformity (ACD) as a complex and heterogeneous disease. ${ }^{1,2}$ The health impact of ACD remains evident, with several studies establishing its severity through health-related quality-of-life measures, where its scores are compa- rable to or even worse than diseases such as heart disease, breast cancer, blindness, and stroke. ${ }^{1-4}$ This is especially the case when the cervical sagittal vertical axis (cSVA) is greater than $4 \mathrm{~cm} .1,3$ Patients with ACD often present with significant disability secondary to the inability to maintain horizontal gaze, abnormal gait, chronic dysphagia, debili-

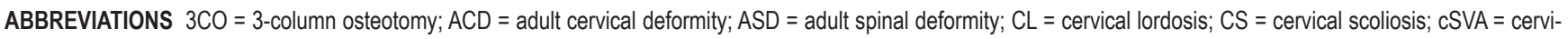
cal SVA; ICU = intensive care unit; LL = lumbar lordosis; LOS = length of stay; NG = nasogastric; $P C O=$ posterior column osteotomy; $P E G$ = percutaneous gastrostomy; PI = pelvic incidence; PT = pelvic tilt; SPO = Smith-Petersen osteotomy; SVA = sagittal vertical axis; TK = thoracic kyphosis; TLS = thoracolumbar scoliosis. SUBMITTED May 12, 2020. ACCEPTED June 12, 2020.

INCLUDE WHEN CITING Published online October 23, 2020; DOI: 10.3171/2020.6.SPINE20826. 
tating neck pain, and/or neurological deficits (myelopathy and radiculopathy). ${ }^{5}$ Fortunately, prior studies have shown that surgical correction of deformity significantly improves disability and pain in ACD patients. ${ }^{6,7}$

There is a growing body of literature describing the treatment and management of ACD patients. ${ }^{8,9}$ The correction of severe and/or fixed (ankylosed) cervical and cervicothoracic deformities often requires bone release to achieve goal correction, with the extent of bone removal dependent on the severity of deformity and on anatomical considerations. Most ACD cases can be adequately corrected with posterior-based approaches using multilevel low-grade osteotomies such as Smith-Petersen osteotomies (SPOs) or high-grade osteotomy such as pedicle subtraction osteotomy. ${ }^{9}$ Due to the invasiveness of these surgical techniques and the frailty of the patient demographic, however, complication rates remain relatively high. The limited studies conducted to assess outcomes in ACD correction report complication rates as high as $43.6 \%$, with this number rising to $56.5 \%$ when evaluating $\mathrm{ACD}$ patients treated via 3 -column osteotomy $(3 \mathrm{CO})$, despite excellent radiographic improvement. ${ }^{10,11}$ The surgical invasiveness coupled with high complication rates can result in extended intensive care unit (ICU) and overall hospital length of stay (LOS). In addition, the ICU is more resource-intensive, and extended LOS in the ICU places a significant burden on healthcare costs for hospitals.

ACD surgery and patient characteristics are very different than the treatment and management of thoracolumbar adult spinal deformity (ASD). A detailed understanding of the ICU course following correction of severe ACD is essential to deliver optimal patient care, and currently the literature surrounding this topic is lacking. Given the paucity of studies concentrating on ICU and hospital LOS for ACD patients, specific modifiable and nonmodifiable risk factors for prolonged ICU and hospital stays have yet to be elucidated. Therefore, in this study we aim to define the average ICU and hospital LOS for ACD surgery and identify risk factors for prolonged LOS. This study also discusses the critical components to ICU care and complication prevention methods for patients undergoing ACD surgery via posterior-based osteotomies.

\section{Methods}

The study protocol received formal approval by the IRB Committee at the University of California, San Francisco.

\section{Study Cohort}

A consecutive cohort of ACD patients who underwent posterior-based osteotomies for deformity correction by the senior author (C.P.A.) from 2010 to 2019 were identified. Inclusion criteria for this study were ACD patients with cSVA of at least $4 \mathrm{~cm}$ and/or cervical kyphosis of $20^{\circ}$ or more. Patients with a diagnosis of acute trauma, cancer, and/or infection were excluded, resulting in a cohort consisting of primary ACD patients, with degenerative, iatrogenic, and inflammatory pathologies. Indications for surgery included severe disability secondary to chronic dysphagia, debilitating neck pain, abnormal gait, progres- sive deformity, and/or neurological deficits (myelopathy and radiculopathy). All surgical treatment utilized posterior-based osteotomies, which comprised either multilevel posterior column osteotomies (PCOs; Cervical Osteotomy Ames grade 2) or 3COs (Cervical Osteotomy Ames grade 5 or 6$).{ }^{9}$

\section{Data Collection}

Data were collected on a retrospective basis from the electronic medical record. Data of interest were patient demographic and clinical variables such as age (years), sex, BMI, smoking status, and presence of preoperative myelopathy and/or weakness. The presence of medical comorbidities was also assessed and included psychiatric disease, stroke, hypertension, diabetes, thyroid disease, gastrointestinal disease, pulmonary disease, cardiac disease, hyperlipidemia, hepatic disease, and renal disease. All radiographic measurements were recorded and performed on standing scoliosis radiographs. Cervical, thoracolumbar, and spinopelvic parameters were gathered both preoperatively and postoperatively to assess for degree of surgical correction. Measurements included cSVA, cervical lordosis (CL), degree of cervical scoliosis (CS), T1-slope, sagittal vertical axis (SVA), thoracic kyphosis (TK), lumbar lordosis (LL), pelvic incidence (PI), pelvic tilt (PT), and thoracolumbar scoliosis (TLS). The methodology for measuring scoliosis radiographs has previously been described. ${ }^{12,13}$ In addition to clinical characteristics, surgical variables were collected and included osteotomy type (PCO vs 3CO), surgical approach, number of segments fused ( $<12$ vs $\geq 12)$, use of decompression, estimated blood loss (liters), operative time (minutes), and presence of a postoperative complication. Adverse outcomes that occurred during patients' hospital stay were tabulated as well. The primary outcomes of the study were ICU and total hospital LOS. ICU LOS was calculated as the number of days spent in the ICU following surgery. Total hospital LOS was defined as the cumulative number of days the patient spent in the hospital from date of admission to date of discharge.

\section{Statistical Analysis}

Continuous variables such as radiographic parameters were compared using paired Student t-tests to assess for significant changes after surgical correction. Given that our outcome variables of ICU and hospital LOS were continuous variables, linear regressions were utilized to identify factors associated with LOS. Univariate linear regressions were initially performed to identify variables of interest, and covariates with a $p$ value $<0.250$ were subsequently incorporated into a multivariate linear regression model to identify independently associated risk factors for extended LOS. All statistical tests were two-tailed, using a p value $<0.050$ for statistical significance. Analysis was performed using the $\mathrm{R}$ statistical program (version 3.6.1, $\mathrm{R}$ Foundation for Statistical Computing).

\section{Results}

\section{Patient Characteristics}

A total of 107 patients were included in the study. A 
summary of demographic, clinical, and surgical characteristics can be found in Table 1 . The mean age for our cohort was 63.5 years, and $61.7 \%$ of patients were female. The average preoperative BMI was $27.8 \mathrm{~kg} / \mathrm{m}^{2}$. A majority of patients $(71.0 \%)$ presented with myelopathy and $40.2 \%$ were found to have weakness on examination. The vast majority of patients $(82.2 \%)$ underwent single-staged posterior-based osteotomies, with only several patients (17.8\%) undergoing staged anterior cervical discectomy. Slightly more than half of the patients $(52.3 \%)$ underwent $3 \mathrm{CO}$ as compared to multilevel PCO in the remainder $(47.7 \%)$. A total of 58 patients $(54.2 \%)$ had 12 or more segments fused, and $83.2 \%$ of all patients underwent decompression. The mean blood loss was $1.1 \mathrm{~L}$ with an average operative time of 273.5 minutes. Overall, 43 patients (40.2\%) experienced a complication (medical, surgical, or neurological), with medical complications being the most prevalent $(26.2 \%)$.

\section{Preoperative and Postoperative Radiographic Parameters}

Table 2 describes the changes in radiographic measurements after surgical correction. Overall, there was significant correction of all cervical parameters: cSVA (6.0 vs $3.6 \mathrm{~cm}, \mathrm{p}<0.001)$, CL $\left(8.2^{\circ}\right.$ vs $\left.-5.3^{\circ}, \mathrm{p}<0.001\right)$, CS $\left(6.5^{\circ}\right.$ vs $\left.2.2^{\circ}, \mathrm{p}<0.001\right)$, and T1-slope $\left(40.2^{\circ}\right.$ vs $\left.34.5^{\circ}, \mathrm{p}<0.001\right)$. There were also reciprocal changes to the distal spine: TK

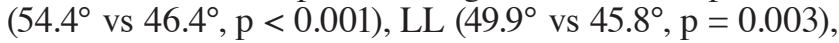
and TLS $\left(13.9^{\circ}\right.$ vs $\left.11.1^{\circ}, \mathrm{p}=0.009\right)$. Cervical, thoracolumbar, and spinopelvic parameters were compared in three distinct cohorts: all patients, patients who underwent highgrade osteotomies, and patients who underwent low-grade osteotomies. When considering patients who underwent $3 \mathrm{CO}$, significant improvement was observed in all cervical measurements: cSVA (6.5 vs $3.8 \mathrm{~cm}, \mathrm{p}<0.001)$, CL $\left(2.3^{\circ}\right.$ [kyphosis] vs $-6.7^{\circ}$ [lordosis], p < 0.001), CS $\left(5.5^{\circ}\right.$ vs $\left.1.8^{\circ}, \mathrm{p}=0.002\right)$, and $\mathrm{T} 1$-slope $\left(48.6^{\circ}\right.$ vs $\left.35.8^{\circ}, \mathrm{p}<0.001\right)$. In addition, there were significant changes in the thoracolumbar spine: TK $\left(64.4^{\circ}\right.$ vs $\left.47.7^{\circ}, \mathrm{p}<0.001\right)$ and LL $\left(52.4^{\circ}\right.$ vs $\left.46.8^{\circ}, \mathrm{p}=0.002\right)$. For the PCO group, significant improvements were observed for all cervical parameters except for T1-slope: cSVA (5.4 vs $3.4 \mathrm{~cm}, \mathrm{p}<0.001)$, CL (14.6 $6^{\circ}$ [kyphosis] vs $-3.9^{\circ}$ [lordosis], $\left.\mathrm{p}<0.001\right), \mathrm{CS}\left(7.4^{\circ}\right.$ vs $\left.2.7^{\circ}, \mathrm{p}=0.004\right)$, and T1-slope $\left(31.4^{\circ}\right.$ vs $\left.33.1^{\circ}, \mathrm{p}=0.347\right)$; there were no significant changes in distal spinal alignment measures. In all three groups, there was no significant change in spinopelvic parameters.

\section{Adverse Events During Hospital Stay Following ACD Surgery}

In Table 3 we report the most significant adverse outcomes observed in patients following ACD correction surgery. Overall, the rates of severe events that occurred during either ICU or hospital stay were low, remaining below 5\% for all complications surveyed. The highest incidence was seen with 5 patients $(4.7 \%)$ who developed pneumonia/pneumonitis, 4 of whom suffered this complication due to an aspiration-related event. A total of 3 patients $(2.8 \%)$ experienced severe dysphagia following surgery, with 2 requiring placement of a nasogastric (NG) or percutaneous gastrostomy (PEG) tube. With regard to
TABLE 1. Overall demographics and clinical data of ACD patients who underwent surgical correction

\begin{tabular}{|c|c|}
\hline Demographic/Clinical Variable & Value \\
\hline No. of patients & 107 \\
\hline Mean age $\pm S D$, yrs & $63.5 \pm 10.1$ \\
\hline \multicolumn{2}{|l|}{ Sex, n (\%) } \\
\hline Male & $41(38.3)$ \\
\hline Female & $66(61.7)$ \\
\hline Mean BMI $\pm \mathrm{SD}, \mathrm{kg} / \mathrm{m}^{2}$ & $27.8 \pm 6.6$ \\
\hline \multicolumn{2}{|l|}{ Smoker, n (\%) } \\
\hline Active & $15(14.0)$ \\
\hline Former & $41(38.3)$ \\
\hline Never & $51(47.7)$ \\
\hline \multicolumn{2}{|l|}{ Preop myelopathy, n (\%) } \\
\hline Yes & $76(71.0)$ \\
\hline No & $31(29.0)$ \\
\hline \multicolumn{2}{|l|}{ Preop weakness, n (\%) } \\
\hline Yes & $43(40.2)$ \\
\hline No & $64(59.8)$ \\
\hline \multicolumn{2}{|l|}{ Osteotomy type, n (\%) } \\
\hline $3 \mathrm{CO}$ & $56(52.3)$ \\
\hline Other & $51(47.7)$ \\
\hline \multicolumn{2}{|l|}{ Surgical approach, n (\%) } \\
\hline Anterior-posterior & $19(17.8)$ \\
\hline Posterior only & $88(82.2)$ \\
\hline \multicolumn{2}{|l|}{ No. of segments fused, $\mathrm{n}(\%)$} \\
\hline$<12$ & $49(45.8)$ \\
\hline$\geq 12$ & $58(54.2)$ \\
\hline \multicolumn{2}{|l|}{ Decompression, n (\%) } \\
\hline Yes & $89(83.2)$ \\
\hline No & $18(16.8)$ \\
\hline \multicolumn{2}{|l|}{ Staged procedure, $\mathrm{n}(\%)$} \\
\hline Yes & $14(13.1)$ \\
\hline No & $93(86.9)$ \\
\hline \multicolumn{2}{|l|}{ Any complication, n (\%) } \\
\hline Yes & $43(40.2)$ \\
\hline No & $64(59.8)$ \\
\hline \multicolumn{2}{|l|}{ Medical complication, n (\%) } \\
\hline Yes & $28(26.2)$ \\
\hline No & $79(73.8)$ \\
\hline \multicolumn{2}{|l|}{ Surgical complication, $\mathrm{n}(\%)$} \\
\hline Yes & $19(17.8)$ \\
\hline No & $88(82.2)$ \\
\hline \multicolumn{2}{|l|}{ Neurological complication, $\mathrm{n}(\%)$} \\
\hline Yes & $18(16.8)$ \\
\hline No & $89(83.2)$ \\
\hline Mean estimated blood loss $\pm \mathrm{SD}, \mathrm{L}$ & $1.1 \pm 1.5$ \\
\hline Mean operative time $\pm S D$, mins & $273.5 \pm 105.5$ \\
\hline Mean ICU LOS \pm SD, days & $2.8 \pm 2.3$ \\
\hline Mean hospital LOS \pm SD, days & $7.9 \pm 3.9$ \\
\hline
\end{tabular}


TABLE 2. Radiographic changes to cervical, thoracolumbar, and spinopelvic parameters in ACD patients who underwent surgical correction, stratified by osteotomy type

\begin{tabular}{|c|c|c|c|c|c|c|}
\hline \multirow{2}{*}{$\begin{array}{c}\text { Radiographic } \\
\text { Parameter }\end{array}$} & \multicolumn{2}{|c|}{ Preop } & \multicolumn{2}{|c|}{ Postop } & \multirow[b]{2}{*}{ Correction } & \multirow[b]{2}{*}{ p Value } \\
\hline & Mean & SD & Mean & SD & & \\
\hline \multicolumn{7}{|l|}{ All patients } \\
\hline $\mathrm{cSVA}(\mathrm{cm})$ & 6.0 & 1.9 & 3.6 & 1.5 & 2.4 & $<0.001$ \\
\hline $\mathrm{CL}\left({ }^{\circ}\right)$ & 8.2 & 26.8 & -5.3 & 17.2 & 13.5 & $<0.001$ \\
\hline $\operatorname{CS}\left({ }^{\circ}\right)$ & 6.5 & 10.8 & 2.2 & 4.3 & 4.3 & $<0.001$ \\
\hline T1-slope $\left({ }^{\circ}\right)$ & 40.2 & 20.4 & 34.5 & 13.0 & 5.7 & $<0.001$ \\
\hline SVA (cm) & 3.0 & 5.6 & 4.0 & 4.9 & 1.0 & 0.186 \\
\hline $\mathrm{TK}\left({ }^{\circ}\right)$ & 54.4 & 19.5 & 46.4 & 16.0 & 8.0 & $<0.001$ \\
\hline $\operatorname{LL}\left({ }^{\circ}\right)$ & 49.9 & 15.1 & 45.8 & 15.4 & 4.1 & 0.003 \\
\hline $\mathrm{PI}\left({ }^{\circ}\right)$ & 56.3 & 14.2 & 55.3 & 13.8 & 1.0 & 0.181 \\
\hline $\mathrm{PT}\left({ }^{\circ}\right)$ & 22.0 & 11.1 & 22.1 & 10.8 & 0.1 & 0.857 \\
\hline $\operatorname{TLS}\left({ }^{\circ}\right)$ & 13.9 & 14.3 & 11.1 & 11.2 & 2.8 & 0.009 \\
\hline \multicolumn{7}{|c|}{ High-grade (3CO) } \\
\hline $\mathrm{cSVA}(\mathrm{cm})$ & 6.5 & 1.9 & 3.8 & 1.6 & 2.7 & $<0.001$ \\
\hline $\mathrm{CL}\left({ }^{\circ}\right)$ & 2.3 & 24.5 & -6.7 & 19.4 & 9.0 & $<0.001$ \\
\hline $\operatorname{CS}\left({ }^{\circ}\right)$ & 5.5 & 9.0 & 1.8 & 3.5 & 3.7 & 0.002 \\
\hline T1-slope $\left({ }^{\circ}\right)$ & 48.6 & 20.7 & 35.8 & 12.7 & 12.8 & $<0.001$ \\
\hline SVA (cm) & 2.9 & 5.3 & 3.6 & 5.0 & 0.7 & 0.377 \\
\hline $\mathrm{TK}\left({ }^{\circ}\right)$ & 64.4 & 15.9 & 47.7 & 11.9 & 16.7 & $<0.001$ \\
\hline $\operatorname{LL}\left({ }^{\circ}\right)$ & 52.4 & 13.7 & 46.8 & 13.8 & 5.6 & 0.002 \\
\hline $\mathrm{PI}\left({ }^{\circ}\right)$ & 55.7 & 12.9 & 54.0 & 12.7 & 1.7 & 0.115 \\
\hline $\mathrm{PT}\left({ }^{\circ}\right)$ & 21.5 & 10.7 & 20.2 & 10.1 & 1.3 & 0.178 \\
\hline $\operatorname{TLS}\left({ }^{\circ}\right)$ & 11.8 & 14.5 & 9.0 & 8.9 & 2.8 & 0.067 \\
\hline \multicolumn{7}{|l|}{ Low-grade (PCO) } \\
\hline cSVA $(\mathrm{cm})$ & 5.4 & 1.7 & 3.4 & 1.5 & 2.0 & $<0.001$ \\
\hline $\mathrm{CL}\left({ }^{\circ}\right)$ & 14.6 & 27.9 & -3.9 & 14.6 & 18.5 & $<0.001$ \\
\hline $\operatorname{CS}\left({ }^{\circ}\right)$ & 7.4 & 12.3 & 2.7 & 5.0 & 4.7 & 0.004 \\
\hline T1-slope $\left({ }^{\circ}\right)$ & 31.4 & 16.0 & 33.1 & 13.3 & 1.7 & 0.347 \\
\hline SVA (cm) & 3.1 & 5.9 & 4.3 & 4.8 & 1.2 & 0.333 \\
\hline $\mathrm{TK}\left({ }^{\circ}\right)$ & 43.7 & 17.4 & 45.1 & 19.4 & 1.4 & 0.136 \\
\hline $\operatorname{LL}\left({ }^{\circ}\right)$ & 47.2 & 16.2 & 44.7 & 17.0 & 2.5 & 0.322 \\
\hline $\mathrm{PI}\left({ }^{\circ}\right)$ & 57.0 & 15.7 & 56.7 & 14.8 & 0.3 & 0.756 \\
\hline $\mathrm{PT}\left({ }^{\circ}\right)$ & 22.7 & 11.6 & 24.0 & 11.4 & 1.3 & 0.110 \\
\hline $\operatorname{TLS}\left({ }^{\circ}\right)$ & 16.2 & 13.8 & 13.5 & 13.0 & 2.7 & 0.061 \\
\hline
\end{tabular}

Boldface type indicates statistical significance.

airway and respiratory status, 4 patients (3.7\%) exhibited symptoms of pulmonary edema and subsequent hypoxemia, with 3 patients requiring prolonged postoperative intubation. One of the 4 patients had acute airway decompensation and required emergency reintubation; despite desperate measures of endotracheal intubation and eventually an emergency cricoidectomy, the patient died from diffuse cerebral hypoxia.

\section{Identifying Risk Factors Associated With LOS}

The mean values for ICU LOS, as well as unstandardized beta coefficients with $p$ values from univariate and multivariate linear regressions for each of our variables,
TABLE 3. Patients with severe adverse events during their hospital stay following ACD correction surgery

\begin{tabular}{lc}
\hline \multicolumn{1}{c}{ Postop Event } & No. of Patients (\%) \\
\hline No. of patients (total) & 107 \\
\hline Aspiration-related complication & $4(3.7)$ \\
\hline Pneumonia/pneumonitis & $5(4.7)$ \\
\hline Airway edema/compromise* & $4(3.7)$ \\
\hline Pulmonary edema/hypoxemia & $4(3.7)$ \\
\hline Delayed extubation & $3(2.8)$ \\
\hline Dyphagia & $3(2.8)$ \\
\hline PEG/NG tube requirement & $2(1.9)$ \\
\hline Hypotension & $3(2.8)$ \\
\hline
\end{tabular}

${ }^{*}$ Required prolonged intubation or reintubation.

can be found in Table 4 . The mean ICU LOS was 2.8 days. Factors that were found to be independently associated with extended LOS in the ICU upon multivariate analysis were sex, number of segments fused, and presence of a complication. Female patients were associated with longer LOS in the ICU (3.0 vs 2.4 days, beta coefficient $1.4, \mathrm{p}=$ $0.004)$ than their male counterparts. Additionally, patients who had 12 or more segments fused (3.5 vs 1.9 days, beta coefficient $1.5, \mathrm{p}=0.002$ ) and the presence of any type of complication (4.0 vs 1.9 days, beta coefficient $2.5, \mathrm{p}=$ 0.017 ) were associated with longer ICU stays.

Table 5 shows the mean values for total hospital LOS, as well as results from the univariate and multivariate linear regressions. The average total hospital LOS was 7.9 days. Multivariate analysis once again demonstrated that sex, number of segments fused, and presence of a complication were independently associated with prolonged total hospital LOS. Female patients were observed to have longer overall hospital stays (8.3 vs 7.3 days, beta coefficient $2.0, \mathrm{p}=0.013$ ). Patients with 12 or more segments fused during surgery had longer overall hospital stays $(9.4$ vs 6.2 days, beta coefficient $2.6, p=0.001$ ). Patients with any complication also had longer hospital stays overall (9.7 vs 6.7 days, beta coefficient 2.4, $\mathrm{p}=0.026$ ).

\section{Discussion}

While the understanding of ACD as a complex surgical disease continues to increase, there is still a relative paucity of literature on the topic as compared to its counterpart in thoracolumbar ASD. Despite this, it is clear that there is a direct correlation between cSVA and worse function and increased disability in ACD patients, especially when the cSVA is greater than $4 \mathrm{~cm} .^{1,3}$ Fortunately, continued advances in surgical technique and the gradual adoption of standardized protocols for treating ACD have led to improved surgical treatments for ACD patients. In addition, multiple studies have shown that patients who undergo surgical correction for ACD gain significant improvement in their deformity-related symptomatology, including relief of debilitating pain.6,7

Despite the fact that patients can benefit tremendously from surgical correction of $\mathrm{ACD}$, these cases often warrant extensive soft-tissue releases, osteotomies, and 
TABLE 4. Average ICU LOS and linear regression models for ACD patients, by demographic, medical, and surgical characteristics

\begin{tabular}{|c|c|c|c|c|c|c|}
\hline \multirow[b]{2}{*}{ Characteristic } & \multicolumn{6}{|c|}{ ICU Stay } \\
\hline & Mean (days) & SD & Beta Coefficient & Univariate $p$ Value & Beta Coefficient & Multivariate $p$ Value \\
\hline \multicolumn{7}{|l|}{ Age (yrs) } \\
\hline$<55$ & 3.7 & 2.6 & Ref & Ref & Ref & Ref \\
\hline $55-70$ & 2.6 & 2.1 & -1.1 & 0.116 & -1.0 & 0.131 \\
\hline$>70$ & 2.8 & 2.4 & -0.8 & 0.296 & -0.8 & 0.283 \\
\hline \multicolumn{7}{|l|}{ Sex } \\
\hline Male & 2.4 & 1.8 & Ref & Ref & Ref & Ref \\
\hline Female & 3.0 & 2.5 & 0.6 & 0.167 & 1.4 & 0.004 \\
\hline \multicolumn{7}{|l|}{$\mathrm{BMI}\left(\mathrm{kg} / \mathrm{m}^{2}\right)$} \\
\hline$<18.5$ & 1.8 & 1.5 & Ref & Ref & Ref & Ref \\
\hline $18.5-25$ & 2.5 & 2.6 & 0.7 & 0.558 & -0.7 & 0.563 \\
\hline $25-30$ & 3.3 & 2.2 & 1.5 & 0.205 & 0.7 & 0.595 \\
\hline$>30$ & 2.6 & 2.0 & 0.9 & 0.475 & 0.7 & 0.548 \\
\hline \multicolumn{7}{|l|}{ Smoking status } \\
\hline Active & 2.3 & 2.2 & Ref & Ref & Ref & Ref \\
\hline Former & 3.2 & 2.7 & 1.0 & 0.161 & 0.7 & 0.300 \\
\hline Never & 2.5 & 1.8 & 0.2 & 0.712 & 0.1 & 0.906 \\
\hline \multicolumn{7}{|l|}{ Comorbidities } \\
\hline \multicolumn{7}{|l|}{ Psychiatric } \\
\hline No & 2.6 & 2.2 & Ref & Ref & & \\
\hline Yes & 3.0 & 2.3 & 0.4 & 0.327 & & \\
\hline \multicolumn{7}{|l|}{ Stroke } \\
\hline No & 2.7 & 2.3 & Ref & Ref & & \\
\hline Yes & 3.4 & 2.1 & 0.7 & 0.279 & & \\
\hline \multicolumn{7}{|l|}{ Hypertension } \\
\hline No & 2.8 & 2.4 & Ref & Ref & & \\
\hline Yes & 2.6 & 2.1 & -0.2 & 0.642 & & \\
\hline \multicolumn{7}{|l|}{ Diabetes } \\
\hline No & 2.8 & 2.3 & Ref & Ref & & \\
\hline Yes & 2.4 & 1.7 & -0.4 & 0.572 & & \\
\hline \multicolumn{7}{|l|}{ Thyroid } \\
\hline No & 2.7 & 2.3 & Ref & Ref & & \\
\hline Yes & 3.0 & 1.5 & 0.3 & 0.668 & & \\
\hline \multicolumn{7}{|l|}{ Gastrointestinal } \\
\hline No & 2.8 & 2.4 & Ref & Ref & & \\
\hline Yes & 2.7 & 2.0 & -0.1 & 0.896 & & \\
\hline \multicolumn{7}{|l|}{ Pulmonary } \\
\hline No & 2.8 & 2.4 & Ref & Ref & & \\
\hline Yes & 2.7 & 1.7 & -0.0 & 0.985 & & \\
\hline \multicolumn{7}{|l|}{ Cardiac } \\
\hline No & 2.7 & 2.3 & Ref & Ref & & \\
\hline Yes & 2.9 & 1.7 & 0.2 & 0.716 & & \\
\hline \multicolumn{7}{|l|}{ Hyperlipidemia } \\
\hline No & 2.9 & 2.3 & Ref & Ref & Ref & Ref \\
\hline Yes & 2.1 & 2.0 & -0.8 & 0.153 & -0.7 & 0.279 \\
\hline \multicolumn{7}{|l|}{ Hepatic } \\
\hline No & 2.8 & 2.3 & Ref & Ref & & \\
\hline Yes & 2.7 & 2.1 & 0.0 & 0.968 & & \\
\hline
\end{tabular}




\section{» CONTINUED FROM PAGE 182}

TABLE 4. Average ICU LOS and linear regression models for ACD patients, by demographic, medical, and surgical characteristics

\begin{tabular}{|c|c|c|c|c|c|c|}
\hline \multirow[b]{2}{*}{ Characteristic } & \multicolumn{6}{|c|}{ ICU Stay } \\
\hline & Mean (days) & SD & Beta Coefficient & Univariate $p$ Value & Beta Coefficient & Multivariate $p$ Value \\
\hline \multicolumn{7}{|c|}{ Comorbidities (continued) } \\
\hline \multicolumn{7}{|l|}{ Renal } \\
\hline No & 2.8 & 2.3 & Ref & Ref & & \\
\hline Yes & 2.0 & 0.8 & -0.8 & 0.501 & & \\
\hline \multicolumn{7}{|l|}{ Preop weakness } \\
\hline No & 3.0 & 2.5 & Ref & Ref & Ref & Ref \\
\hline Yes & 2.4 & 1.7 & -0.6 & 0.185 & -0.4 & 0.413 \\
\hline \multicolumn{7}{|l|}{ Abnormal gait } \\
\hline No & 2.7 & 2.3 & Ref & Ref & & \\
\hline Yes & 2.8 & 2.3 & 0.1 & 0.873 & & \\
\hline \multicolumn{7}{|l|}{ Kyphosis } \\
\hline$<20^{\circ}$ & 2.7 & 2.6 & Ref & Ref & & \\
\hline$\geq 20^{\circ}$ & 2.9 & 1.8 & 0.2 & 0.593 & & \\
\hline \multicolumn{7}{|l|}{ cSVA (cm) } \\
\hline$<4$ & 2.4 & 2.0 & Ref & Ref & Ref & Ref \\
\hline $4-6$ & 2.4 & 1.8 & 0.1 & 0.937 & 0.5 & 0.488 \\
\hline $6-8$ & 3.1 & 2.7 & 0.7 & 0.876 & 0.7 & 0.339 \\
\hline$>8$ & 3.4 & 2.6 & 1.1 & 0.234 & 0.6 & 0.492 \\
\hline \multicolumn{7}{|l|}{ Osteotomy type } \\
\hline High-grade & 3.3 & 2.3 & Ref & Ref & Ref & Ref \\
\hline Low-grade & 2.1 & 2.0 & -1.2 & 0.007 & 0.1 & 0.811 \\
\hline \multicolumn{7}{|l|}{ Segments fused } \\
\hline$<12$ & 1.9 & 1.6 & Ref & Ref & Ref & Ref \\
\hline$\geq 12$ & 3.5 & 2.4 & 1.6 & $<0.001$ & 1.5 & 0.002 \\
\hline \multicolumn{7}{|l|}{ Approach } \\
\hline Combined & 3.7 & 2.7 & Ref & Ref & Ref & Ref \\
\hline Posterior & 2.5 & 2.1 & -1.2 & 0.034 & -0.5 & 0.385 \\
\hline \multicolumn{7}{|l|}{ Laminectomy } \\
\hline No & 3.3 & 2.7 & Ref & Ref & & \\
\hline Yes & 2.6 & 2.2 & -0.6 & 0.275 & & \\
\hline \multicolumn{7}{|l|}{ Any complication } \\
\hline No & 1.9 & 1.5 & Ref & Ref & Ref & Ref \\
\hline Yes & 4.0 & 2.7 & 2.0 & $<0.001$ & 2.5 & 0.017 \\
\hline \multicolumn{7}{|l|}{ Medical complication } \\
\hline No & 2.3 & 1.7 & Ref & Ref & Ref & Ref \\
\hline Yes & 4.1 & 3.0 & 1.8 & $<0.001$ & -0.4 & 0.674 \\
\hline \multicolumn{7}{|l|}{ Surgical complication } \\
\hline No & 2.6 & 2.3 & Ref & Ref & Ref & Ref \\
\hline Yes & 3.6 & 1.9 & 1.0 & 0.08 & -3.7 & 0.116 \\
\hline \multicolumn{7}{|c|}{ Neurological complication } \\
\hline No & 2.6 & 2.3 & Ref & Ref & Ref & Ref \\
\hline Yes & 3.6 & 1.9 & 1.0 & 0.07 & 3.6 & 0.109 \\
\hline
\end{tabular}

Boldface type indicates statistical significance. 
TABLE 5. Average hospital LOS and linear regression models for ACD patients by demographic, medical, and surgical characteristics

\begin{tabular}{|c|c|c|c|c|c|c|}
\hline \multirow[b]{2}{*}{ Characteristic } & \multicolumn{6}{|c|}{ Hospital Stay } \\
\hline & Mean (days) & SD & Beta Coefficient & Univariate $p$ Value & Beta Coefficient & Multivariate $p$ Value \\
\hline \multicolumn{7}{|l|}{ Age (yrs) } \\
\hline$<55$ & 8.6 & 2.7 & Ref & Ref & & \\
\hline $55-70$ & 7.6 & 3.7 & -1.0 & 0.421 & & \\
\hline$>70$ & 8.6 & 4.9 & 0.0 & 0.986 & & \\
\hline \multicolumn{7}{|l|}{ Sex } \\
\hline Male & 7.3 & 3.1 & Ref & Ref & Ref & Ref \\
\hline Female & 8.3 & 4.3 & 1.0 & 0.179 & 2.0 & 0.013 \\
\hline \multicolumn{7}{|l|}{ BMI $\left(\mathrm{kg} / \mathrm{m}^{2}\right)$} \\
\hline$<18.5$ & 5.3 & 1.5 & Ref & Ref & Ref & Ref \\
\hline $18.5-25$ & 8.9 & 5.0 & 3.7 & 0.074 & 1.0 & 0.645 \\
\hline $25-30$ & 8.2 & 3.7 & 2.9 & 0.154 & 0.8 & 0.688 \\
\hline$>30$ & 7.1 & 2.7 & 1.8 & 0.369 & 0.6 & 0.780 \\
\hline \multicolumn{7}{|l|}{ Smoking status } \\
\hline Active & 8.1 & 3.3 & Ref & Ref & & \\
\hline Former & 8.9 & 5.1 & 0.8 & 0.485 & & \\
\hline Never & 7.1 & 2.6 & -0.9 & 0.411 & & \\
\hline \multicolumn{7}{|l|}{ Comorbidities } \\
\hline \multicolumn{7}{|l|}{ Psychiatric } \\
\hline No & 7.6 & 3.5 & Ref & Ref & Ref & Ref \\
\hline Yes & 8.6 & 4.5 & 1.0 & 0.189 & 0.6 & 0.397 \\
\hline \multicolumn{7}{|l|}{ Stroke } \\
\hline No & 7.8 & 3.9 & Ref & Ref & & \\
\hline Yes & 8.9 & 4.1 & 1.1 & 0.343 & & \\
\hline \multicolumn{7}{|l|}{ Hypertension } \\
\hline No & 8.1 & 4.2 & Ref & Ref & & \\
\hline Yes & 7.7 & 3.5 & -0.5 & 0.516 & & \\
\hline \multicolumn{7}{|l|}{ Diabetes } \\
\hline No & 7.9 & 4.0 & Ref & Ref & & \\
\hline Yes & 8.4 & 3.4 & 0.6 & 0.612 & & \\
\hline \multicolumn{7}{|l|}{ Thyroid } \\
\hline No & 7.8 & 3.7 & Ref & Ref & & \\
\hline Yes & 8.7 & 5.4 & 0.9 & 0.456 & & \\
\hline \multicolumn{7}{|l|}{ Gastrointestinal } \\
\hline No & 7.9 & 3.6 & Ref & Ref & & \\
\hline Yes & 8.1 & 4.4 & 0.2 & 0.781 & & \\
\hline \multicolumn{7}{|l|}{ Pulmonary } \\
\hline No & 8.0 & 4.1 & Ref & Ref & & \\
\hline Yes & 7.6 & 3.1 & -0.4 & 0.639 & & \\
\hline \multicolumn{7}{|l|}{ Cardiac } \\
\hline No & 7.8 & 4.0 & Ref & Ref & & \\
\hline Yes & 8.7 & 3.3 & 0.9 & 0.403 & & \\
\hline \multicolumn{7}{|l|}{ Hyperlipidemia } \\
\hline No & 8.0 & 3.9 & Ref & Ref & & \\
\hline Yes & 7.6 & 4.0 & -0.5 & 0.652 & & \\
\hline \multicolumn{7}{|l|}{ Hepatic } \\
\hline No & 7.9 & 3.6 & Ref & Ref & & \\
\hline Yes & 9.0 & 6.9 & 1.1 & 0.455 & & \\
\hline
\end{tabular}




\section{» CONTINUED FROM PAGE 184}

TABLE 5. Average hospital LOS and linear regression models for ACD patients by demographic, medical, and surgical characteristics

\begin{tabular}{|c|c|c|c|c|c|c|}
\hline \multirow[b]{2}{*}{ Characteristic } & \multicolumn{6}{|c|}{ Hospital Stay } \\
\hline & Mean (days) & SD & Beta Coefficient & Univariate $p$ Value & Beta Coefficient & Multivariate $p$ Value \\
\hline \multicolumn{7}{|c|}{ Comorbidities (continued) } \\
\hline \multicolumn{7}{|l|}{ Renal } \\
\hline No & 7.9 & 3.9 & Ref & Ref & & \\
\hline Yes & 8.3 & 2.2 & 0.3 & 0.869 & & \\
\hline \multicolumn{7}{|l|}{ Preop weakness } \\
\hline No & 8.2 & 3.7 & Ref & Ref & & \\
\hline Yes & 7.6 & 4.2 & -0.6 & 0.474 & & \\
\hline \multicolumn{7}{|l|}{ Abnormal gait } \\
\hline No & 7.9 & 3.7 & Ref & Ref & & \\
\hline Yes & 8.0 & 4.2 & 0.1 & 0.878 & & \\
\hline \multicolumn{7}{|l|}{ Kyphosis } \\
\hline$<20^{\circ}$ & 7.9 & 3.8 & Ref & Ref & & \\
\hline$\geq 20^{\circ}$ & 8.2 & 4.2 & 0.3 & 0.703 & & \\
\hline \multicolumn{7}{|l|}{ cSVA (cm) } \\
\hline$<4$ & 7.3 & 3.1 & Ref & Ref & Ref & Ref \\
\hline $4-6$ & 7.0 & 3.2 & -0.3 & 0.806 & 0.7 & 0.551 \\
\hline $6-8$ & 8.5 & 4.7 & 1.2 & 0.3705 & 1.5 & 0.245 \\
\hline$>8$ & 10.2 & 3.7 & 2.9 & 0.055 & 2.5 & 0.083 \\
\hline \multicolumn{7}{|l|}{ Osteotomy type } \\
\hline High-grade & 8.7 & 3.7 & Ref & Ref & Ref & Ref \\
\hline Low-grade & 7.1 & 3.9 & -1.6 & 0.029 & -0.2 & 0.783 \\
\hline \multicolumn{7}{|l|}{ Segments fused } \\
\hline$<12$ & 6.2 & 2.7 & Ref & Ref & Ref & Ref \\
\hline$\geq 12$ & 9.4 & 4.1 & 3.3 & $<0.001$ & 2.6 & 0.001 \\
\hline \multicolumn{7}{|l|}{ Approach } \\
\hline Combined & 9.8 & 3.9 & Ref & Ref & Ref & Ref \\
\hline Posterior & 7.5 & 3.8 & -2.3 & 0.018 & -0.9 & 0.286 \\
\hline \multicolumn{7}{|l|}{ Laminectomy } \\
\hline No & 9.0 & 3.6 & Ref & Ref & Ref & Ref \\
\hline Yes & 7.7 & 3.9 & -1.3 & 0.203 & -0.5 & 0.600 \\
\hline \multicolumn{7}{|l|}{ Any complication } \\
\hline No & 6.7 & 3.2 & Ref & Ref & Ref & Ref \\
\hline Yes & 9.7 & 4.1 & 3.0 & $<0.001$ & 2.4 & 0.026 \\
\hline \multicolumn{7}{|l|}{ Medical complication } \\
\hline No & 7.2 & 3.4 & Ref & Ref & Ref & Ref \\
\hline Yes & 10.1 & 4.3 & 2.9 & $<0.001$ & 0.4 & 0.763 \\
\hline \multicolumn{7}{|l|}{ Surgical complication } \\
\hline No & 7.8 & 4.0 & Ref & Ref & & \\
\hline Yes & 8.4 & 3.1 & 0.6 & 0.550 & & \\
\hline \multicolumn{7}{|c|}{ Neurological complication } \\
\hline No & 7.8 & 4.0 & Ref & Ref & & \\
\hline Yes & 8.4 & 3.2 & 0.5 & 0.589 & & \\
\hline
\end{tabular}

Boldface type indicates statistical significance. 
long construct fusions in order to achieve the desired results. ${ }^{10,11,14}$ Similar to prior studies, our results demonstrate that posterior-based osteotomies and soft-tissue releases are able to achieve excellent surgical correction. ${ }^{8,10-12,15-17}$ When looking at improvement in radiographic measurements across all patients, we observed that both cervical and thoracolumbar measurements significantly improved without changes in spinopelvic parameters. However, on subgroup analysis of $3 \mathrm{CO}$ and $\mathrm{PCO}$, only $3 \mathrm{CO}$ was associated with significant changes in T1-slope and thoracolumbar parameters, highlighting the power of $3 \mathrm{CO}$ s for $\mathrm{ACD}$ correction. It is also important to note that the subset of patients undergoing $3 \mathrm{CO}$ on average had greater preoperative cSVA (neck translation), CL (more lordosis), T1slope, and TK than PCO cases. Conversely, PCO cases had abnormal cSVA to a lesser degree with lower CL (worse kyphosis). This trend highlights how surgical approach as well as osteotomy type and location depend heavily on the specific type of ACD being treated. ${ }^{9}$ For ACD with abnormal cSVA $(>4 \mathrm{~cm})$ due to an abnormally high T1-slope $\left(>30^{\circ}\right)$, the deformity stems mainly from a cervicothoracic or upper thoracic deformity. In such cases, a $3 \mathrm{CO}$ is warranted to augment the abnormally high T1-slope. For positive cSVA mainly due to cervical kyphosis in the subaxial spine (normal T1-slope), multilevel PCO should be sufficient. ${ }^{6,11,18-20}$

Given the paucity of comprehensive literature for ACD surgery, it is important to continue investigating clinical questions such as what risk factors may prolong patient ICU and hospital LOS after ACD surgery. The average ICU and total hospital LOS in our cohort were 2.8 days and 7.9 days, respectively. For both ICU and total hospital stay, we observed that many variables were significant on univariate analysis. However, given the high degree of association and likelihood of confounders within these variables, it was important to create a comprehensive multivariate model as well to identify independent associations. ${ }^{21}$ To this end, we incorporated not only variables that were significant on univariate analysis, but also all variables with a $\mathrm{p}$ value $<0.250$. The risk factors ultimately found to be independently associated with both longer ICU and hospital LOS were female sex, 12 or more segments fused during surgery, and the presence of a complication. Two of the 3 identified variables (fusion levels and the presence of a complication) are potentially modifiable in practice with goals of minimizing the need for prolonged ICU and hospital LOS.

The longer LOS stay for patients with 12 or more segments fused is likely explained by the fact that number of segments fused serves as a proxy for complexity and invasiveness of the surgery being performed. Often, the complexity is dictated by the deformity severity and need for particular surgical strategies. These results are consistent with a 2019 study by Horn et al. and the International Spine Study Group (ISSG) to predict extended operative time and length of inpatient stay in ACD corrective surgery, which also showed greater number of segments fused (7 or more in their study) and complications to be associated with extended LOS. ${ }^{22}$ Thus, it is essential to choose the appropriate surgical strategy and counsel your patients accordingly.
As identified in this study, the presence of a complication was also independently associated with longer ICU (4.0 vs 1.9 days) and hospital (9.7 vs 6.7 days) LOS. Presence of complication was significant in multivariate models for both ICU and total hospital stay, with large beta coefficients of 2.6 and 2.4, respectively, illustrating the large impact (approximately 2.5-day increase in LOS in each regression model) that complication profile had on LOS. Interestingly, when complication type was isolated, none of the specific types of complications (medical, surgical, or neurological) remained significant in the multivariate model; neurological complication was the closest in trending toward significance $(\mathrm{p}=0.109)$, with a large beta coefficient of 3.6 indicating a large increase in LOS for patients with neurological complications versus those without. The results of our study corroborate prior cervical spine studies (nondeformity cohorts) that highlight overall postoperative complication rates as predictive of increased LOS. ${ }^{23,24}$

Perioperative complications are especially important to consider in ACD patients as they are generally a much frailer subset of patients, especially when compared to patients being treated for degenerative disease or thoracolumbar ASD. This increased frailty stems from a multitude of factors, including malnourishment and subsequent cachexia, which can occur as a result of their cervical deformity. Multiple studies have measured the vulnerability of ACD patients using metrics such as the cervical deformity frailty index and the Quality of Life in Swallowing Disorders questionnaire, which has shown a high level of preoperative swallowing dysfunction in ACD cohorts. ${ }^{25,26}$ In particular, a study by Miller et al. showed that cervical deformity patients exhibited a mean preoperative cervical deformity frailty index of 0.44 (severe frailty) and highlighted a significant association between increasing patient frailty and postoperative outcomes, including complication rate, in ACD patients. ${ }^{25}$ As a result of their multisystem impairments, patients with a high degree of frailty demonstrate diminished physiological response to surgery-related stressors. ${ }^{27}$ Therefore, ACD patients who undergo deformity correction are often at higher risk of perioperative complications. The significance of complication status in the multivariate model is not surprising, but emphasizes the importance of patient optimization in the preoperative setting, morbidity prevention, and appropriate complication management. It is crucial to understand the optimal perioperative care of ACD patients following deformity correction. As mentioned earlier, neurological deficits have the most impact on need for prolonged ICU and hospital LOS. For ACD correction, most deficits are a result of isolated nerve root weakness (such as C5 and C8 palsy) rather than spinal cord injury. The most effective way to minimize neurological risk is to utilize intraoperative neuromonitoring, but its effectiveness for nerve root monitoring in ACD has not been studied.

An additional aim of this study was to identify patients who suffered severe adverse events during their ICU or hospital stays, to better inform the need for ICU management and continuous pulse monitoring postoperatively. While the overall rate of complications in this study was relatively high (40.2\%), we saw a low incidence of severe 
adverse events $(<5 \%)$ during patients' ICU and hospital stays. Almost all ACD patients who undergo large corrections warrant ICU care for a variety of reasons: hemodynamic instability (requiring rapid transfusions), blood pressure augmentation, close neurological monitoring, adequate pain control, and airway management. Blood pressure augmentation (especially in the presence of neuromonitoring changes) is critical for ensuring that spinal cord perfusion is sufficient, and this can be immediately titrated according to changes in the neurological examination. In this study, 3 patients (2.8\%) suffered from hypotension during their hospitalization. Frequent neurological examination is also critical for being able to detect further complications such as a compressive hematoma resulting in spinal cord injury, especially following 3CO. Unlike the management of thoracolumbar ASD patients, one of the most important aspects of caring for ACD patients after surgery is safe and proper airway management. ACD patients are at extremely high risk for airway obstruction secondary to manipulation of the neck with concurrent tracheal and palatal edema (due to prolonged prone positioning and large fluid shifts during surgery). ${ }^{28,29}$ Intubating these patients while in extremis is extremely difficult and, at times, not possible without emergency cricothyrotomy, especially following cervical fusion in which there is limited neck extension. ${ }^{29}$ A total of 3 patients $(2.8 \%)$ were kept intubated for a prolonged period secondary to concern for airway edema, and 1 patient $(0.9 \%)$ required emergency reintubation during their postoperative hospital stay. Unfortunately, that 1 patient could not be reintubated (via endotracheal routes) and required an emergency bedside cricothyrotomy due to compromised airway. Despite all attempts, the patient suffered global hypoxic ischemia with rapid deterioration, resulting in death. As such, management of the airway is essential and needs to be carefully discussed with the anesthesia and ICU teams, with sufficient plans put into place prior to the operation. In addition to fragile respiratory status, ACD patients are at high risk for dysphagia and aspiration; this risk is present even without approaching the spine anteriorly. ${ }^{30-32}$ In our study, 3 patients $(2.8 \%)$ experienced dysphagia, with 2 requiring placement of an NG or PEG tube to provide sufficient nutrition. There were also 5 patients $(4.7 \%)$ who experienced pneumonia/pneumonitis as a result of aspiration-related complications.

The importance of this study lies in our ability to identify higher-risk populations of patients that may warrant extra attention for risk stratification and minimization of postoperative morbidity. As the risk factors identified in this study are both nonmodifiable and modifiable risk factors, the clinical impact of our results stems from the ability to understand which patients may be at higher risk when performing ACD correction surgery, so they can be monitored accordingly. Ultimately, this can develop into standardized protocols for management of patients based on perioperative characteristics. In 2019, Ayrian et al. published the results of their collaborative study between neuroanesthesiologists and spine surgeons, which sought to implement a strict perioperative management protocol for complex spine surgery across all spine regions (cervical, thoracic, lumbar, and multiple regions). ${ }^{33}$ The aim of the study was to determine if following a rigorous and methodical perioperative protocol could decrease total hospital and ICU LOS after elective complex spine surgery. Their study showed that implementation of a standard spine surgery protocol significantly reduced total hospital and ICU LOS for their patients, with no significant differences in the rates of postoperative complications between the two groups. However, as similar protocols start to become adopted more widely at hospitals, it is important to distinguish between degenerative disease, thoracolumbar ASD, and ACD as separate disease entities that may warrant unique procedures and protocols. By better understanding the clinical course of ACD correction surgery, we can begin to devise similar protocols on a larger scale that are specific to correction of cervical deformities; this will allow us to provide a safer and more beneficial operative course for patients.

The limitations of this study are primarily in its retrospective design. One of the drawbacks of a retrospective analysis is the possibility of the results being skewed based on selection bias. All patients were treated at a tertiary care spine center that receives referrals from many different regions, so the patient population may not accurately model the general demographic for this disease. Additionally, given the retrospective nature of the study, it is possible that confounding variables remain in our analysis that might have been explained by additional variables or measurements that were not collected. In addition, ACD remains a far less common disease than thoracolumbar ASD, and as such sample sizes and patient cohorts are significantly smaller, oftentimes hampering our ability to conduct meaningful analysis. However, the strength of our study also relates to the size of our overall patient cohort as a single-institution experience, which minimizes intersurgeon variability in technique and protocol. Our sample size of 107 patients is also relatively large when compared to similar studies in the literature for ACD, of which most are multiinstitutional studies. Regardless, it is vital that further prospective studies be conducted for validation of our findings, and that additional analysis should follow as more patient data become available.

\section{Conclusions}

Surgical correction of rigid ACD can be challenging and, in most cases, requires the use of posterior-based osteotomies including multilevel SPO and 3CO. While these surgical techniques can achieve excellent radiographic improvement, they remain invasive and can be associated with high complication rates as well as prolonged ICU and hospital LOS. Our study suggests that sex, number of segments fused, and complication profile are independently associated risk factors for prolonged LOS in both the ICU and the hospital. For both hospital and ICU LOS, our results showed that female patients, as well as those with 12 or more segments fused or any complication during/ following ACD correction surgery, had significantly longer LOS. While these variables encompass both modifiable and nonmodifiable risk factors, patients meeting these criteria may warrant extra attention postoperatively. Complications following ACD correction surgery, however, 
could benefit from continued refinement of surgical technique and postoperative management as well as additional studies profiling clinical outcomes in these patients. It is important that these findings be validated in prospective studies as we continue to learn more about surgical outcomes following ACD correction.

\section{References}

1. Scheer JK, Tang JA, Smith JS, et al. Cervical spine alignment, sagittal deformity, and clinical implications: a review. $J$ Neurosurg Spine. 2013;19(2):141-159.

2. Smith JS, Line B, Bess S, et al. The health impact of adult cervical deformity in patients presenting for surgical treatment: comparison to United States population norms and chronic disease states based on the EuroQuol-5 dimensions questionnaire. Neurosurgery. 2017;80(5):716-725.

3. Pierce KE, Alas H, Brown AE, et al. PROMIS physical health domain scores are related to cervical deformity severity. J Craniovertebr Junction Spine. 2019;10(3):179-183.

4. Tang JA, Scheer JK, Smith JS, et al. The impact of standing regional cervical sagittal alignment on outcomes in posterior cervical fusion surgery. Neurosurgery. 2012;71(3):662-669.

5. Griegel-Morris P, Larson K, Mueller-Klaus K, Oatis CA. Incidence of common postural abnormalities in the cervical, shoulder, and thoracic regions and their association with pain in two age groups of healthy subjects. Phys Ther. 1992;72(6):425-431.

6. Deviren V, Scheer JK, Ames CP. Technique of cervicothoracic junction pedicle subtraction osteotomy for cervical sagittal imbalance: report of 11 cases. J Neurosurg Spine. 2011;15(2):174-181.

7. Etame AB, Wang AC, Than KD, et al. Outcomes after surgery for cervical spine deformity: review of the literature. Neurosurg Focus. 2010;28(3):E14.

8. Ames CP, Smith JS, Eastlack R, et al. Reliability assessment of a novel cervical spine deformity classification system. $J$ Neurosurg Spine. 2015;23(6):673-683.

9. Ames CP, Smith JS, Scheer JK, et al. A standardized nomenclature for cervical spine soft-tissue release and osteotomy for deformity correction: clinical article. J Neurosurg Spine. 2013;19(3):269-278.

10. Smith JS, Ramchandran S, Lafage V, et al. Prospective multicenter assessment of early complication rates associated with adult cervical deformity surgery in 78 patients. Neurosurgery. 2016;79(3):378-388.

11. Smith JS, Shaffrey CI, Lafage R, et al. Three-column osteotomy for correction of cervical and cervicothoracic deformities: alignment changes and early complications in a multicenter prospective series of 23 patients. Eur Spine J. 2017;26(8):2128-2137.

12. Ames CP, Blondel B, Scheer JK, et al. Cervical radiographical alignment: comprehensive assessment techniques and potential importance in cervical myelopathy. Spine (Phila Pa 1976). 2013;38(22)(suppl 1):S149-S160.

13. Ames CP, Smith JS, Scheer JK, et al. Impact of spinopelvic alignment on decision making in deformity surgery in adults: a review. J Neurosurg Spine. 2012;16(6):547-564.

14. Koller H, Ames C, Mehdian H, et al. Characteristics of deformity surgery in patients with severe and rigid cervical kyphosis (CK): results of the CSRS-Europe multi-centre study project. Eur Spine J. 2019;28(2):324-344.

15. Passias PG, Horn SR, Raman T, et al. The impact of osteotomy grade and location on regional and global alignment following cervical deformity surgery. J Craniovertebr Junction Spine. 2019;10(3):160-166.

16. Smith JS, Klineberg E, Shaffrey CI, et al. Assessment of surgical treatment strategies for moderate to severe cervical spi- nal deformity reveals marked variation in approaches, osteotomies, and fusion levels. World Neurosurg. 2016;91:228-237.

17. Passias PG, Jalai CM, Lafage V, et al. Primary drivers of adult cervical deformity: Prevalence, variations in presentation, and effect of surgical treatment strategies on early postoperative alignment. Neurosurgery. 2018;83(4):651-659.

18. Kim KT, Lee SH, Son ES, et al. Surgical treatment of "chin-on-pubis" deformity in a patient with ankylosing spondylitis: a case report of consecutive cervical, thoracic, and lumbar corrective osteotomies. Spine (Phila Pa 1976). 2012;37(16):E1017-E1021.

19. Tokala DP, Lam KS, Freeman BJC, Webb JK. C7 decancellisation closing wedge osteotomy for the correction of fixed cervico-thoracic kyphosis. Eur Spine J. 2007;16(9):14711478.

20. Mummaneni PV, Dhall SS, Rodts GE, Haid RW. Circumferential fusion for cervical kyphotic deformity. J Neurosurg Spine. 2008;9(6):515-521.

21. Wang H, Peng J, Wang B, et al. Inconsistency between univariate and multiple logistic regressions. Shanghai Jingshen Yixue. 2017;29(2):124-128.

22. Horn SR, Passias PG, Bortz CA, et al. Predicting extended operative time and length of inpatient stay in cervical deformity corrective surgery. J Clin Neurosci. 2019;69:206-213.

23. Guan J, Karsy M, Schmidt MH, et al. Multivariable analysis of factors affecting length of stay and hospital charges after single-level corpectomy. J Clin Neurosci. 2017;44:279-283.

24. De la Garza-Ramos R, Goodwin CR, Abu-Bonsrah N, et al. Prolonged length of stay after posterior surgery for cervical spondylotic myelopathy in patients over 65 years of age. $J$ Clin Neurosci. 2016;31:137-141.

25. Miller EK, Ailon T, Neuman BJ, et al. Assessment of a novel adult cervical deformity frailty index as a component of preoperative risk stratification. World Neurosurg. 2018;109:e800-e806.

26. Iyer S, Kim HJ, Bao H, et al. Cervical deformity patients have baseline swallowing dysfunction but surgery does not increase dysphagia at 3 months: results from a prospective cohort study. Global Spine J. 2019;9(5):532-539.

27. Segreto FA, Passias PG, Brown AE, et al. The influence of surgical intervention and sagittal alignment on frailty in adult cervical deformity. Oper Neurosurg (Hagerstown). 2020;18(6):583-589.

28. Li H, Huang Y, Shen B, et al. Multivariate analysis of airway obstruction and reintubation after anterior cervical surgery: a retrospective cohort study of 774 patients. Int J Surg. 2017;41:28-33.

29. Debkowska MP, Butterworth JF, Moore JE, et al. Acute postoperative airway complications following anterior cervical spine surgery and the role for cricothyrotomy. J Spine Surg. 2019;5(1):142-154.

30. Cameron K, Lawless MH, Conway R, et al. Risk factors for dysphagia following a cervical fusion in a trauma population. Cureus. 2018;10(10):e3489.

31. Bakhsheshian J, Mehta VA, Liu JC. Current diagnosis and management of cervical spondylotic myelopathy. Global Spine J. 2017;7(6):572-586.

32. Radcliff KE, Koyonos L, Clyde C, et al. What is the incidence of dysphagia after posterior cervical surgery? Spine (Phila Pa 1976). 2013;38(13):1082-1088.

33. Ayrian E, Sugeir SH, Arakelyan A, et al. Impact of a perioperative protocol on length of ICU and hospital stay in complex spine surgery. J Neurosurg Anesthesiol. Published online August 9, 2019. doi:10.1097/ANA.0000000000000635

\section{Disclosures}

Dr. Deviren reports being a consultant to NuVasive, Medicrea, AlphaTec, Seaspine, and Biomet. Dr. Ames reports receiving roy- 
alties from Stryker, Biomet Zimmer Spine, DePuy Synthes, NuVasive, Next Orthosurgical, K2M, and Medicrea; being a consultant to DePuy Synthes, Medtronic, Medicrea, and K2M; receiving research support from Titan Spine, DePuy Synthes, and ISSG; being on the editorial board of Operative Neurosurgery; receiving grant funding from SRS; being on the executive committee of ISSG; and being a director of Global Spine Analytics.

\section{Author Contributions}

Conception and design: Lau, Joshi, Deviren, Ames. Acquisition of data: Lau, Joshi, Deviren, Ames. Analysis and interpretation of data: Lau, Joshi, Ames. Drafting the article: Joshi. Critically revising the article: Lau, Joshi, Ames. Reviewed submitted version of manuscript: Lau, Joshi, Haddad, Ames. Approved the final version of the manuscript on behalf of all authors: Lau. Statistical analysis: Lau, Joshi. Study supervision: Lau, Deviren, Ames.

\section{Supplemental Information}

Previous Presentations

The findings from this paper were given as an oral presentation at the AANS/CNS Spine Summit in Las Vegas, Nevada, on Sunday, March 8, 2020.

\section{Correspondence}

Darryl Lau: University of California, San Francisco, CA. darryl.lau@ucsf.edu. 